\author{
Military Technical College \\ Kobry El-Kobbah, \\ Cairo, Egypt
}

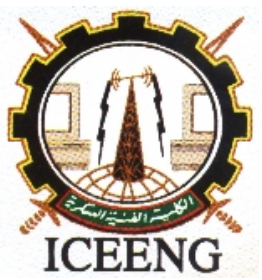

\author{
$8^{\text {th }}$ International Conference \\ on Electrical Engineering \\ ICEENG 2012
}

\title{
Pileup Recovery Algorithms For Digital GAMMA RAY SPECTROSCOPY
}

\author{
Imbaby I. Mahmoud ${ }^{1}$, Mohamed S. El_Tokhy ${ }^{1}$ and Hussein A. Konber ${ }^{2}$ \\ ${ }^{1}$ Engineering Department, NRC, Atomic Energy Authority, Inshas, Cairo, Egypt \\ imbabyisma@yahoo.com,engtokhy@gmail.com
}

${ }^{2}$ Electrical Engineering Department, Al Azhar University, Nasr City, Cairo

\begin{abstract}
This paper presents algorithms for overcoming a common problem of gamma ray spectroscopy, namely the peak pileup recovery problem. Three different approaches are studied and evaluated within a spectroscopy system. The algorithms are evaluated by the means of parameters error and fitting error calculations. The first approach is a direct search based on Nelder-Mead technique without any derivatives in order to find the local minimum points. A Gaussian shape in conjunction with the peak height and its position of each pulse are used to construct the pulse. So, the main pulse parameters such as peak amplitude, position and width can be determined. The second algorithm is based on the nonlinear least square method. This approach has accuracy of recovering the original pulses with mean square error of $4.7306 \times 10^{-12}$. In this paper another technique is tried. This technique which is proposed as third algorithm is based on a maximum peak search method combined with the first derivative method to determine peak position of each pulse. Comparison among these approaches is conducted in terms of parameters errors. The pulse parameters have been calculated and compared with the actual one. The second approach shows the best accuracy, for determining peak height and position, but the width parameter scored the highest error.
\end{abstract}

\section{KEYWORDS}

Peak Pileup, Tail Pileup, Least-Square Fitting, Nelder-Mead Modified Simplex.

\section{INTRODUCTION}

Spectroscopic gamma-ray detectors are used for many research, industrial, medical and homeland-security applications [1-4]. The advantages of a digital system for gamma ray spectroscopy in comparison with a classical analog system are reflected in the possibilities of implementation of complex algorithms and simple and rapid modification of algorithms used for signal processing. Using these systems the highest quality of measurements is achieved at both low and high counting rates with various radiation detectors [5]. Thallium-doped sodium iodide $(\mathrm{NaI}(\mathrm{Tl})$ ) scintillation crystals coupled to photomultiplier tubes provide medium resolution spectral data about the surrounding environment. These effects are hardware- 
dependent and have strong effects on the radioisotopic identification capability of $\mathrm{NaI}(\mathrm{Tl})$ based systems. Pulse pileup distortion is a common problem for radiation spectroscopy measurements at high counting rates [4-6]. Moreover, pileup is one of the most delicate problems of any spectrometric method that is related to the extraction of the correct information out of the experimental spectra. In many applications as much as $80 \%$ of information can be lost due to the effects of dead time and pulse pileup [7]. The effects of pulse pileup in applications of nuclear techniques include the following issues. Imposing a fundamental limit on detector throughput (and therefore source intensity), decreased spectral accuracy and resolution, as peaks in the energy spectrum spread, reduced peak-to-valley ratios due to false detection of pulses, and causing significant detector dead time in the system [7]. Therefore without a correction on the response function of the detector system incorrect physical data are obtained from an analysis of measured spectra [8]. The deconvolution methods are widely applied in various fields of data processing and various approaches can be employed [8]. On one hand it must decompose completely the overlapping peaks while preserving as much as possible their heights, positions, areas and widths. The objective of this paper is to study different algorithms implementing these methods. So, we discuss the performances of three different pileup recovery algorithms; direct search, least square fitting, and first derivative combined with maximum peak search algorithms. Our primary focus is the analysis of pileup signals collected from detector systems to obtain high accuracy of the spectroscopy. The influence of white Gaussian noise on the recovery performance in the direct search and least square fitting algorithms is furthermore discussed. Pileup correction of gamma ray spectroscopy using these three different algorithms is studied. These algorithms have the advantages of decomposition of multiple overlapping events into their original peaks. This paper is organized as follows: Section 2 presents the spectroscopy system. The more interesting characteristics of pileup recovery algorithms, and registration or rejection of the peak height are represented in Section 3. Comparison between the three algorithms and discussion are summarized in Section 4 and we terminate our study by a briefly discussion and stating some important conclusions that we noted from our obtained results.

\section{SYSTEM COMPONENT}

In this system, the components of the system for evaluation of different pileup recovery algorithms are described. It contains the following elements; ${ }^{137} \mathrm{Cs}$ point source, scintillation detector, amplifier, digital system and connection to a desktop personal computer (PC). An 1.5 inches $\times 7.5$ inches $\mathrm{NaI}(\mathrm{TI})$ scintillation detector is used to detect the radiation signal from $\mathrm{Cs}^{137}$ point source. This detector is connected to amplifier through coaxial cable which in turn connected to the PC through digital scope. The digital scope used for signal digitization with sampling frequency of $16 \mathrm{MS} / \mathrm{s}$. MATLAB environment is used to perform background corrections, pileup, and spectrum evaluation.

\section{Evaluation of Pileup Recovery Algorithms}

\subsection{General Pileup Recovery Using Deconvolution [9]}

The differential pulse height spectrum that is recorded from any radiation detector is the convolution of its inherent response function and the energy distribution of the incident radiation. The deconvolution is sometimes used for the process of resolving or decomposing a set of overlapping peaks into their separate components by different techniques. Pulse pileup is an important issue for radiation spectroscopy applications of scintillation detectors, since the maximum counting rate is limited by pulse pileup. Radiation particles are emitted from a source following the interval distribution as shown below $[4,9,10]$

$f(\Delta t) d t=\lambda_{n} e^{-\lambda_{n} \Delta t} d t$

where $\lambda_{\mathrm{n}}, \mathrm{t}$, and $\mathrm{dt}$ denote the true average emission rate of the radiation, the time interval between the current time pulse stamp and the relative origin of the time axis (that is 
conveniently the occurrence time of the previous pulse), and the infinitely small time interval between $t$ and $t+d t$. A certain amount of light collection time is necessary for good energy resolution.

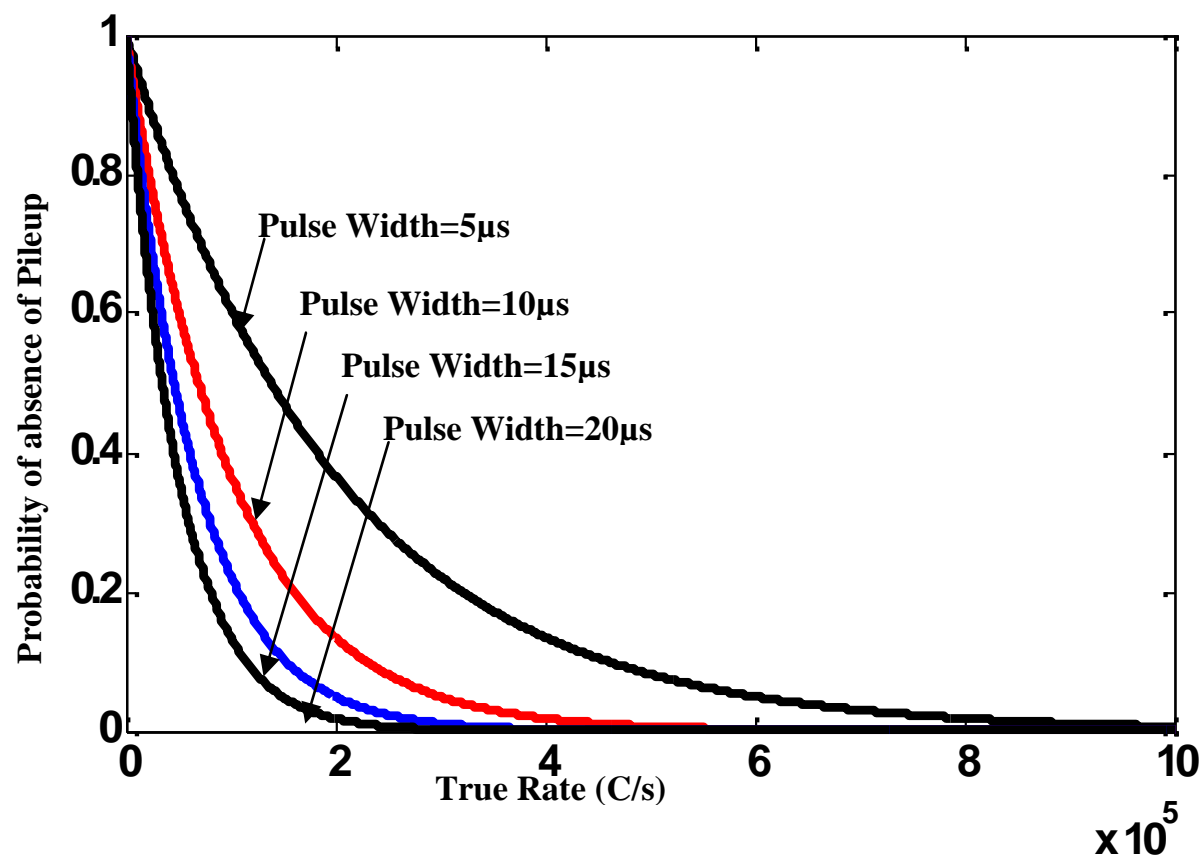

Figure 1. Probability of absence of pileup with the true counting rate at different pulse widths

The probability $\mathrm{P}(\mathrm{N})$ for one pulse to occur without piling up with subsequent pulses may be estimated by

$$
P(\Delta t>\tau)=e^{-\lambda_{n} \tau}
$$

where $\tau$ is the first pulse width. Figure 1 shows the probability curve of absence of pileup against true rate at different pulse widths. As illustrated in this figure for applications where counting rate can be kept very low, pulse pileup distortion is not a problem. Also, the probability of absence of pileup decreases with increases the width of the first pulse. From the figure, we observe the dependency of pileup probability on both pulse width and true rate. In the following, three different algorithms for the pileup recovery are investigated.

\subsection{Direct Search Method}

Here, we are interested in resolving or decomposing a set of overlapping peaks into their separate components. Nelder-Mead modified simplex technique is used for this purpose. Direct search is a method for solving optimization problems that does not require any information about the gradient of the objective function. As opposed to more traditional optimization methods that use information about the gradient or higher derivatives to search for an optimal point, a direct search algorithm searches a set of points around the current point, looking for one where the value of the objective function is lower than the value at the current point. We can use direct search to solve problems for which the objective function is not differentiable, or even continuous [11]. Direct search is used to describe sequential examination of trial solutions involving comparison of each trial solution with the "best" obtained up to that time together with a strategy for determining what the next trial solution will be [12]. It remains popular because of their simplicity, flexibility, and reliability. Examples of direct search methods are the Nelder-Mead Simplex method, Hooke and Jeeves' pattern search, the box method, and Dennis and Torczon`s Parallel Direct Search Algorithm (PDS) [13]. The simplex algorithm is one of the 
earliest and best known optimization algorithms. This algorithm solves the linear programming problems. The algorithm moves along the edges of the polyhedron defined by the constraints, from one vertex to another, while decreasing the value of the objective function at each step. A modified simplex method for finding a local minimum of a function of several variables has been devised by Nelder and Mead [14]. The Nelder-Mead modified simplex algorithm succeeds in obtaining a good reduction in the function value using a relatively small number of function evaluations. This method finds the minimum of a function of several variables [15], starting at an initial estimate. This is generally referred to as unconstrained nonlinear optimization. Search methods that use only function evaluations (the simplex search of Nelder-Mead) are most suitable for problems that are very nonlinear or have a number of discontinuities. Since, it is a direct search method it does not use numerical or analytic gradients [11]. Despite its widespread use, essentially no theoretical results have been proved explicitly for the Nelder-Mead algorithm [16]. For two variables, a simplex is a triangle, and the method is a pattern search that compares function values at the three vertices of a triangle. The worst vertex, where the function value is largest, is rejected and replaced with a new vertex. A new triangle is formed and the search is continued. The process generates a sequence of triangles (which might have different shapes), for which the function values at the vertices get smaller and smaller. The size of the triangles is reduced and the coordinates of the minimum point are found. This algorithm is stated using one time the term simplex (a generalized triangle in $N$ dimensions) and other time Nelder-Mead to find the minimum of a function of $N$ variables. It is effective and computationally compact [14]. In other words, if $\mathrm{n}$ is the length of vector, $\mathrm{x}$, a simplex in $\mathrm{n}$-dimensional space is characterized by the $n+1$ distinct vectors that are its vertices. In two-space, a simplex is a triangle; in threespace, it is a pyramid. At each step of the search, a new point in or near the current simplex is generated. The function value at the new point is compared with the function's values at the vertices of the simplex. Usually, one of the vertices is replaced by the new point, giving a new simplex. This step is repeated until the diameter of the simplex is less than the specified tolerance [11]. The MATLAB implementation of this algorithm finds both local minimum and maximum for a function without derivative [11]. Figure 2 depicts the algorithm for pileup recovery peaks using Nelder-Mead direct search method. As shown in this figure, this algorithm is applied to two simulated overlapping peaks. Moreover, white Gaussian noise is added to these peaks to test the accuracy of the algorithm. Nelder-Mead algorithm is essentially a way of organizing and optimizing the changes in parameters to shorten the time required to fit function to the required degree of accuracy. The most general way of fitting any model to a set of data is the iterative method. Consequently, iterative fit is performed. Iterative methods proceed in the following general way as depicted in Fig. 3. This figure is illustrated as in the following [15]

1) Selects a model for the data;

2) First guesses of all the non-linear parameters are made;

3) A computer program computes the model and compares it to the data set, calculating a fitting error;

4) If the fitting error is greater than the required fitting accuracy, the program systematically changes one or more of the parameters and loops back around to step 3 .

The pulse parameters; peak position, height and width are determined using this Nelder-Mead modified simplex algorithm. The pulse shape is constructed using its peak shape as Gaussian shape [17-20]. Consequently, the original peaks are recovered. Finally, the recovered peaks are registered as described in the following subsection. This algorithm is applied to two simulated overlapping peaks as depicted in Fig. 4 (a). Two nonlinear parameters; peak position and width are determined using this algorithm. A Gaussian shape in conjunction with the peak and its position of each pulse are used to construct each pulse. The recovered two peaks with the input pileup peaks are shown in Fig. 4 (b). However, these two peaks are illustrated separately in Fig. 4 (c). The pulse parameters for the two recovered signals are calculated as depicted in Table 1. These parameters are the pulse position, maximum amplitude, FWHM (full width peak maximum) and the pulse area. The obtained results showed that peak parameters were able to be recovered within $0.24 \%$ deviations. Figures 5-6 depict the relation between the percent 
parameters errors (he difference between the actual parameters and the parameters of the best-fit model) against percent fitting error (the RMS difference between the model and the data) for both the first and second peak, respectively. The values of these errors are determined as explained in Fig. 3. A basic assumption of any curve fitting operation is that the fitting error and the parameter error are minimized. The variability of the fitting error is caused by random small variations in the first guesses, rather than by random noise in the signal. The variability of the fitting error is caused by random small variations in the first guesses, and by noise in the signal.

1) Input Pileup Signal Containing Two Overlapping Peaks

2) Adding White Gaussian Noise to the Input Signal

3) Applying Nelder-Mead Technique

A) Do Iterative Fit Routine

B) Determine the Parameters of the Noisy Input Signal

4) Apply Recovery Peaks Routine

5) Detect Illusive Pulses (If Exist)

6) Register Actual Peaks

Figure 2. Pileup recovery using the Nelder-Mead direct search algorithm

Table 1. Average measured parameters for double peaks overlapping

\begin{tabular}{|c|c|c|c|c|}
\hline Estimated Parameters & Max. Peak & Position & Pulse Width & Area \\
\hline $1^{\text {st }}$ Peak & 4.9840 & 99.8769 & 100.2060 & 527.0110 \\
\hline $2^{\text {nd }}$ Peak & 2.9954 & 250.0082 & 200.0181 & 636.6072 \\
\hline
\end{tabular}




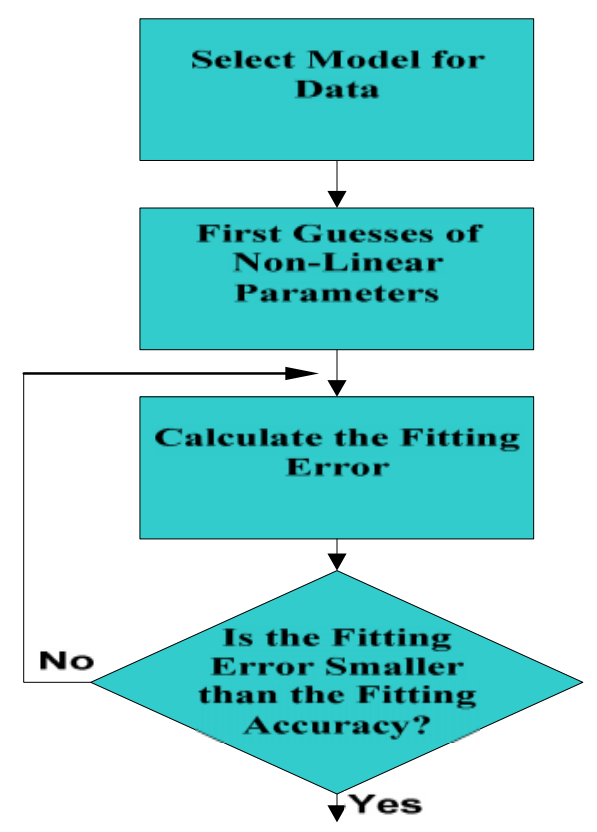

Figure 3. Flowchart of the iterative methodology

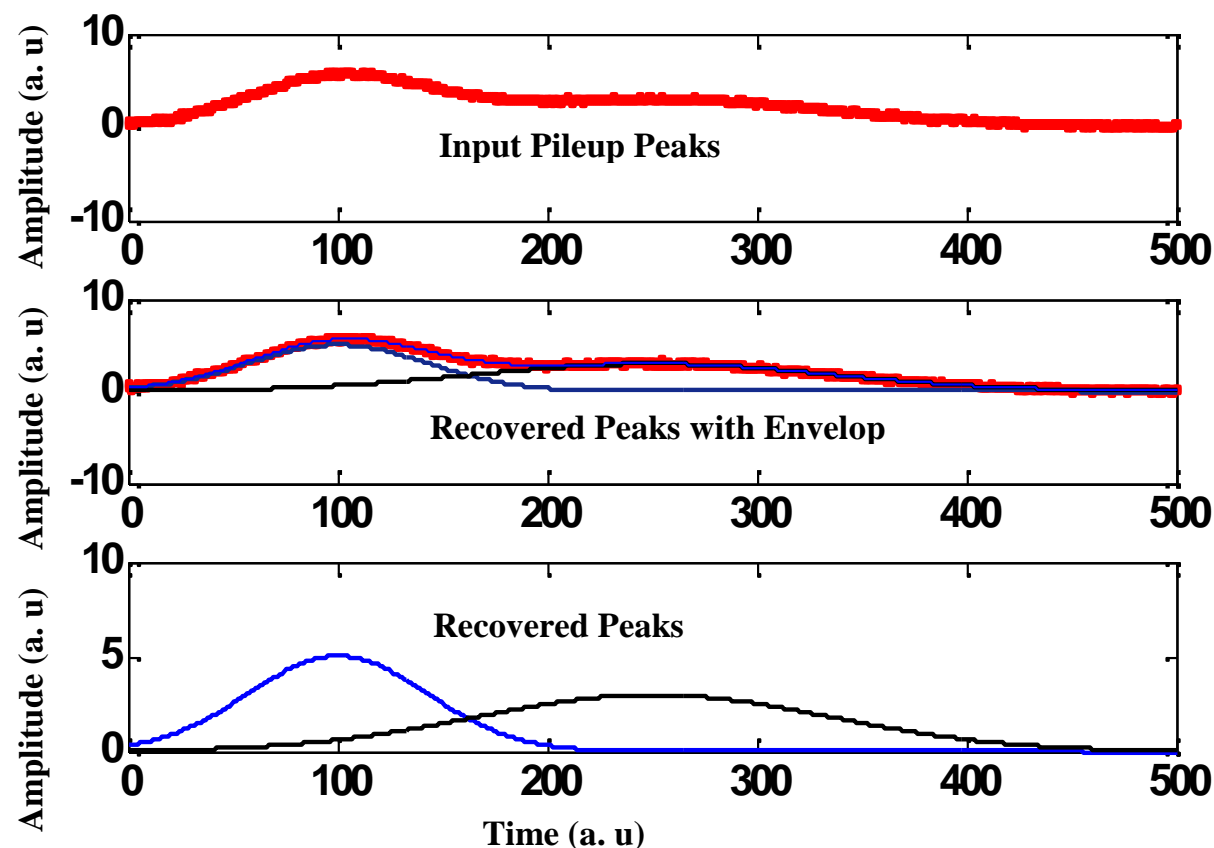

Figure 4. Peaks recovery using direct search algorithm 


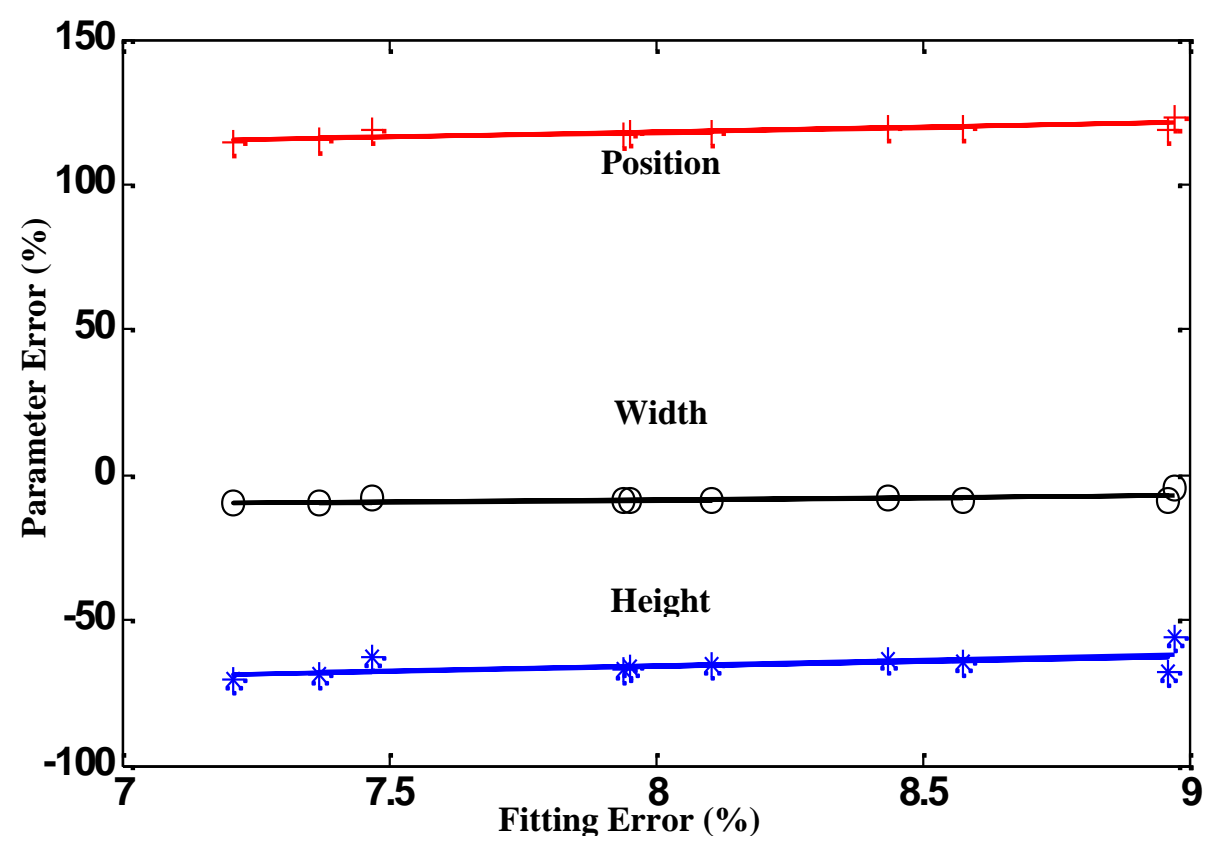

Figure 5. Percent fitting error against percent parameter error for first peak

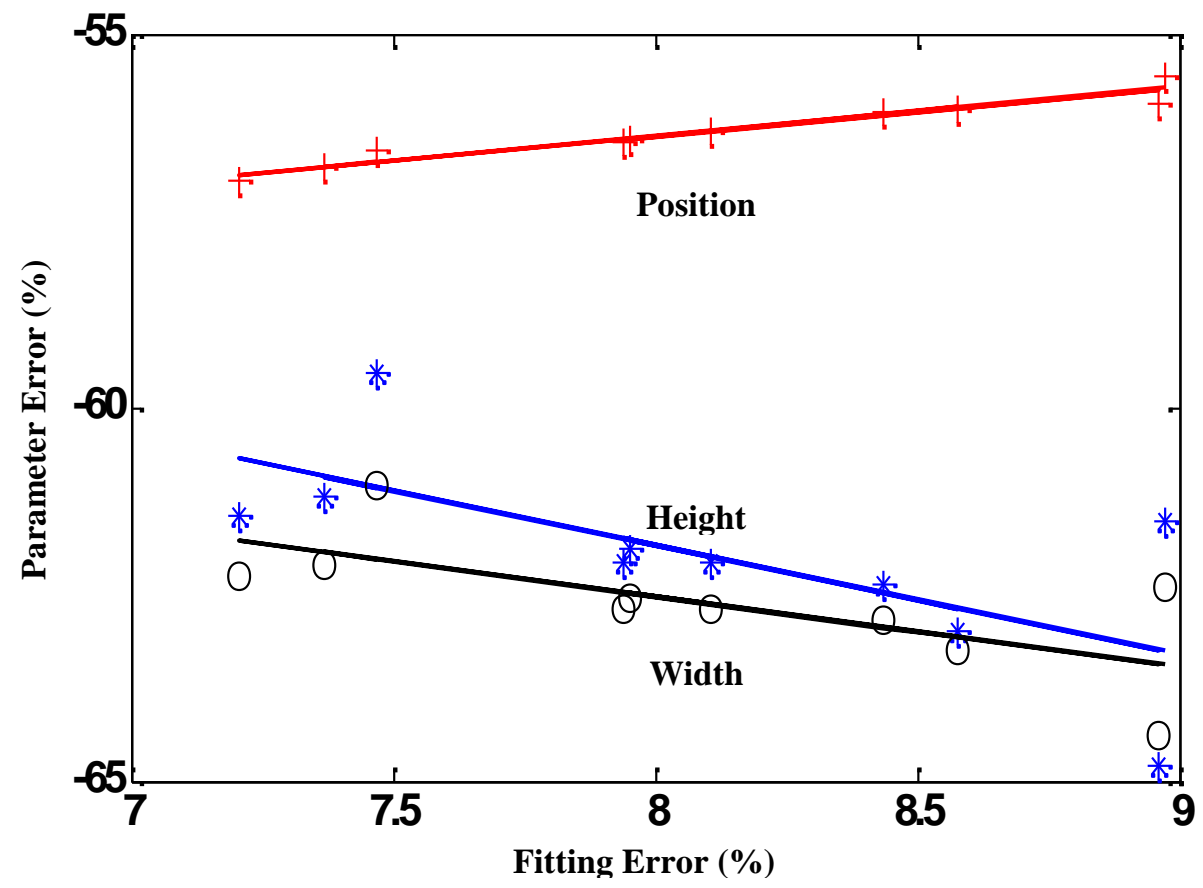

Figure 6. Percent fitting error against percent parameter error for second peak

\subsection{Least Square Fitting Algorithm}

Traditionally, least squares curve fitting is used because it is easy to implement. It provides effective results in many fields of applications like signal processing, and noise cancellation. For resolving pileup overlapping peaks, the standard least-square technique is used [21]. The least squares analysis is used to fit a set of $m$ observations with a model that is non-linear in $n$ unknown parameters $(m>n)$. Solution of least square fitting problem is an iterative process, whose convergence speed is problem-dependent. This technique is employed for initial guessing 
of peak parameters. These parameters are peak height, position, and width. The objective of curve fitting is to find a mathematical equation that describes a set of data. A simple and improved algorithm to resolve overlapped asymmetric pulses into its component peaks using nonlinear least square fitting method is reported in [10, 22, 23]. Figure 7 illustrates this algorithm. Considering voltage waveform, two overlapping peaks are characterized by the following equation after applying the fitting procedure

$V(T)=a_{1} e^{(-(T-b 1) / c 1)}+a_{2} e^{\left(-\left(T-b_{2}\right) / c_{2}\right)}$

where $\mathrm{V}(\mathrm{T}), \mathrm{a}_{1,2}, \mathrm{~b}_{1,2}$, and $\mathrm{c}_{1,2}$ denote the voltage waveform of two overlapping peaks as a function of time, the amplitude, position and the width of the first and second recovered pulses, respectively. For signal recovery step, the overlapped peaks were assumed to be a convolution of its component peaks. It was characterized by Gaussian shape [17-20]. Therefore, the obtained coefficients are fitted to match the simulated two peaks. Consequently, the two overlapping peaks are recovered. As a final stage the recovered peaks can be rejected or accepted as described in the following subsection. Figure 8 shows both the simulated two overlapping peaks and the fitting Gaussian shape. The two recovered peaks with the original overlapping peaks are shown in Fig. 9. The calculated mean square error between the input pileup and recovered peaks of this algorithm was found to be $4.7306 \times 10^{-12}$.

\section{1) Input Pileup Signal Containing Two Overlapping Peaks}

2) Adding White Gaussian Noise to the Input Signal

3) Applying Nonlinear Least Square Fitting Technique

A) Do Iterative Fit with Gaussian model

B) Determine the Parameters of the Noisy Input Signal

4) Apply Recovery Peaks Routine

5) Detect Illusive Pulses (If Exist)

6) Register Actual Peaks

Figure 7. Pileup recovery using nonlinear least square fitting algorithm 


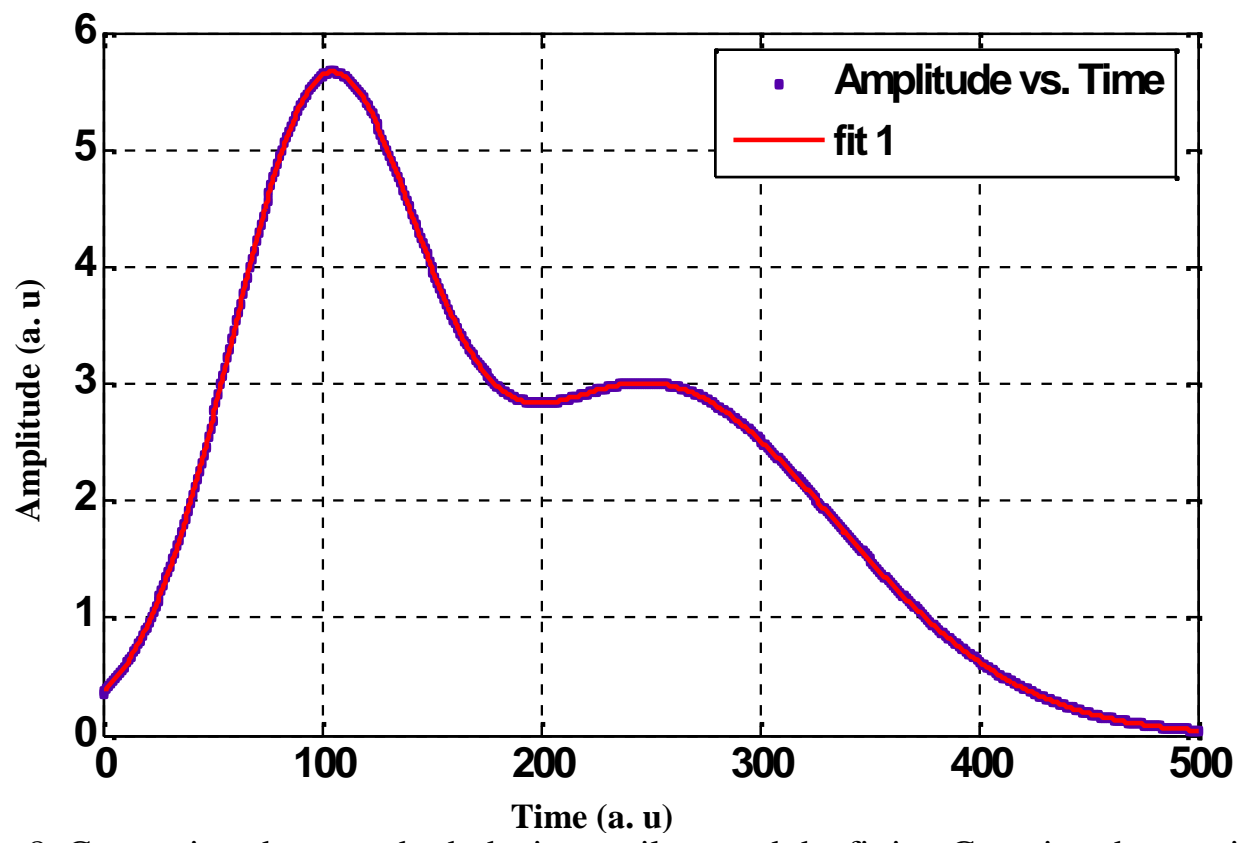

Figure 8. Comparison between both the input pileup and the fitting Gaussian shape using the nonlinear least square method.

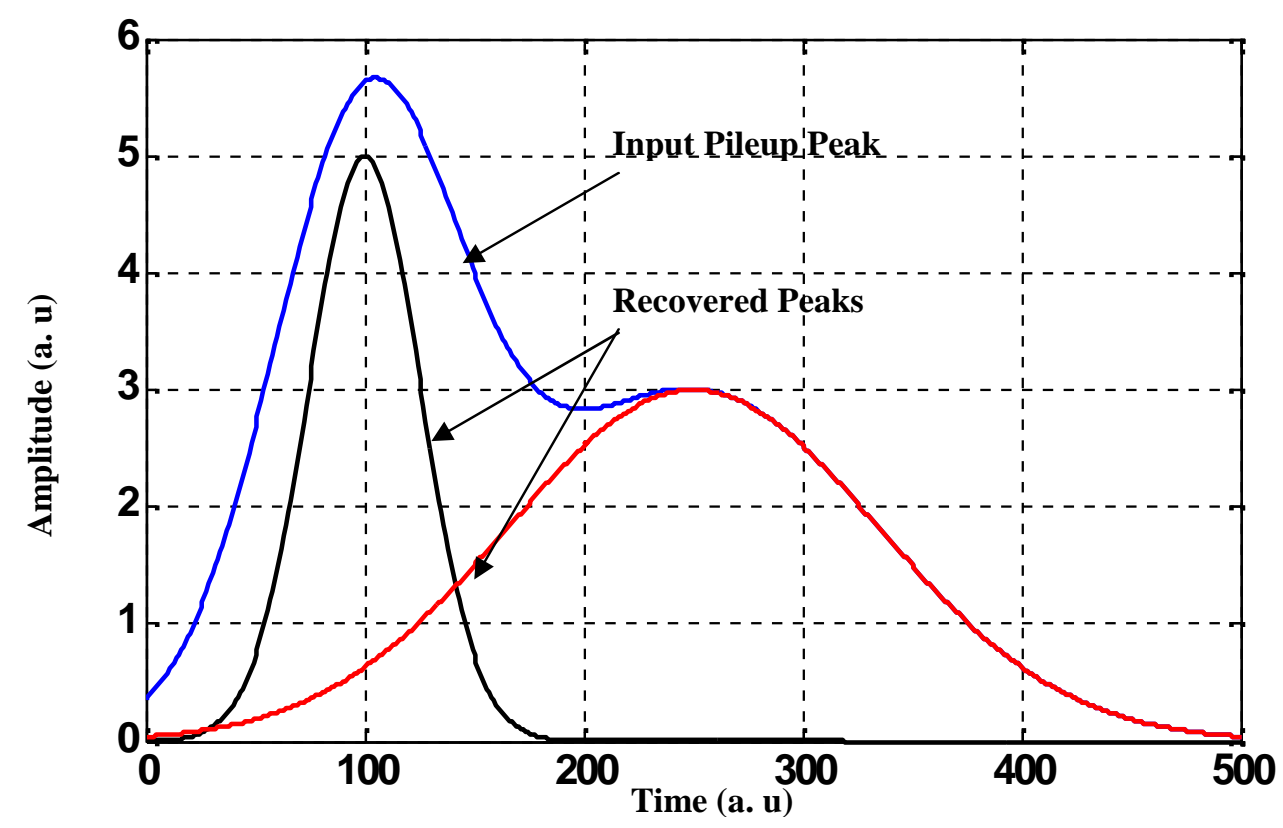

Figure 9. The input pileup peaks and the recovered two overlapping peaks.

\subsection{First Derivative and Maximum Peak Search Algorithm}

The third algorithm for pileup recovery is a proposed one that is presented in Fig. 10. The detection of the peak and determination of the peak position can be realized in successive steps, independent of each other. This algorithm is based on first derivative of peak overlapping pulses, and a MATLAB routine implementing the maximum peak search algorithm [24, 25, 26]. The differentiation of signals is used to facilitate the detection and location of partially overlapped Gaussian peaks in a multi-component signal [27]. First derivative method has been used because it has the capability of spectral discrimination [28-29]. For peak search process, 
most peak search algorithms for $\gamma$-ray spectrometry are based on digital filters. The maximum peak is determined using the maximum peak search routine. This routine search and find local maxima in the overlapping peaks. Furthermore, this algorithm can be applied using FIR filter. This filter represents the number of passes of the small running average filter in order to get rid of small peaks. For signal recovery step, the overlapped peak was assumed to be a convolution of its component peaks. It was characterized by Gaussian shape as in [17-20]. Therefore, the obtained coefficients are fitted with the simulated input peaks. Consequently, the two overlapping peaks are recovered. As a final stage in Fig. 10, the recovered peaks can be rejected or accepted as described in the following subsection. Figure 11 (a) shows the pulse derivative amplitude against time for overlapping peaks. Consequently, the first derivative of both peaks is shown in Fig. 11 (a). From this figure, one can predict the shape of each peak by recalling that the derivative is the slope of the original signal. The derivative of the signal is positive as the signal slopes up. However, the derivative of the signal is negative as the signal slopes down. If a signal has zero slopes, its derivative is zero. Consequently, the location of the maximum in a peak signal can be computed precisely by computing the location of the zero-crossing in its first derivative. The peak amplitude of the signal is determined by using a maximum peak search algorithm as depicted in Fig. 11 (b). The simulated overlapping peak signals are depicted in Fig. 12 (a). The recovered two peaks with the original overlapping peaks are illustrated in Fig. 12 (b). However, these two signals are shown separately in Fig. 12 (c).

\section{1) Input Pileup Signal Containing Two Overlapping Peaks}

2) Adding White Gaussian Noise to the Input Signal

3) Do the $1^{\text {st }}$ Derivative Process to Determine Peaks Positions

4) Do Maximum Peak Search Routine to Determine Peaks Heights

5) Develop a Gaussian pulses shape using the obtained parameters

6) Apply Recovery Peaks Routine

7) Detect Illusive Pulses (If Exist)

\section{8) Register Actual Peaks}

Figure 10. Pileup recovery using first derivative and maximum peak search algorithm 


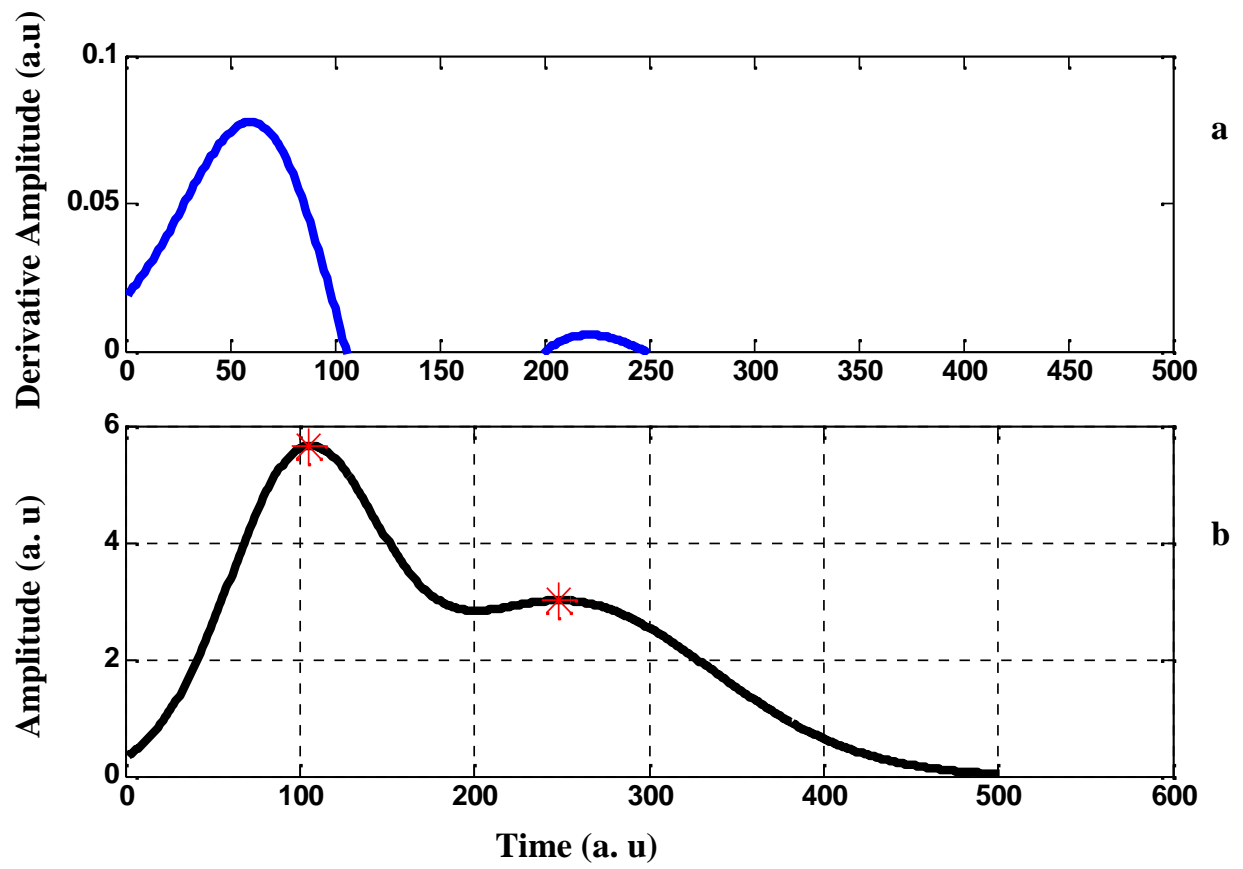

Figure 11. a) First derivative result of double peak overlapping signal and b) Maximum peak amplitudes of the signal.

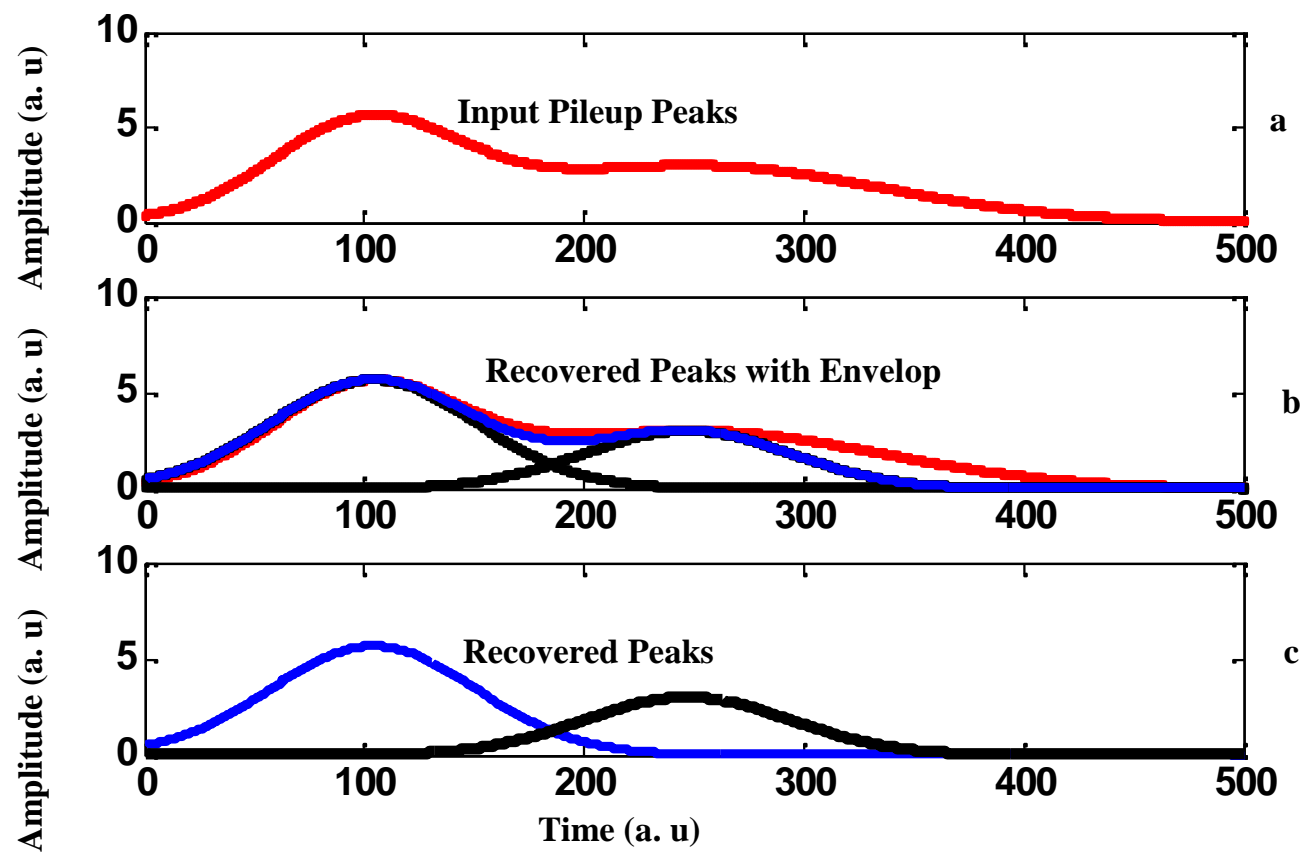

Figure 12. Peaks recovery using first derivative with maximum peak search method

\subsection{Gaussian Noise Handling}

Moreover, white Gaussian noise is added to the overlapping peaks to test the accuracy of the algorithms. The effect of white Gaussian noise on the three algorithms is studied. The variation between signal-noise-ratio (SNR) and the maximum error between both the recovered peak and original peak is depicted in Figure 13. As shown in this figure, the third algorithm is robust enough to noise at low SNR. However, the first algorithm introduces better results at high SNR. Consequently, signal preprocessing is essential to achieve low error with the first algorithm. 
However, the pulse width error is the main reason of high error that originated with the second algorithm. As a final conclusion, the third algorithms give us satisfactory results in noisy environment. In contrast, the first and second algorithms show high sensitivity with noise. Therefore, noise cancellation is essential with the both first and second algorithms.

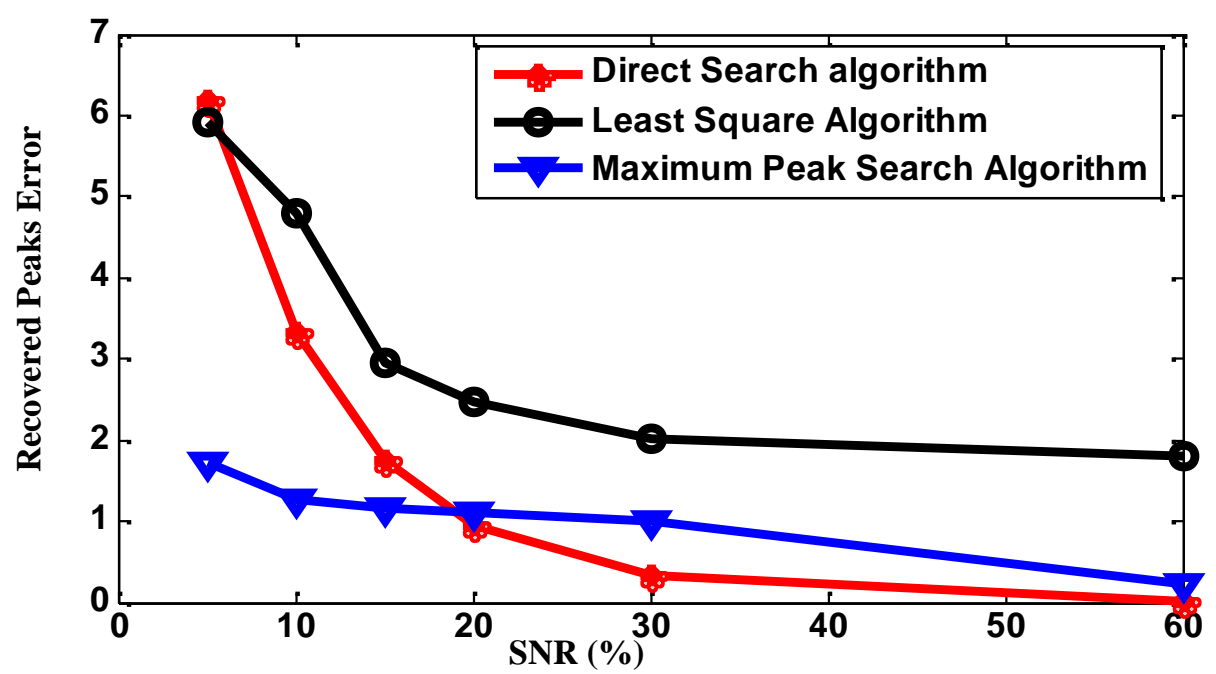

Figure 13. Recovered peaks error against SNR for the algorithms

\subsection{Registration and Rejection of the Peak Height}

Obviously, the occurrence time of the pulse is defined to be the time point where the leading edge voltage reaches the threshold [9]. Naturally, the time interval between two adjacent pulses can be defined as the difference of the occurrence time for these two pulses. Based on the above definitions, it is clear that pulse pileup occurs whenever the time interval of two adjacent pulses is less than the pulse width of the first pulse $[4,9]$. On other hand, for pileup to be avoided, the interval following each pulse must be greater than the effective pulse width. The recovered peak is registered if the difference between the two overlapping peaks is grater than the pulse width otherwise the peak is neglected as follow

$\mathrm{T}_{2}-\mathrm{T}_{1}>\frac{F W H M_{1}}{2}+\frac{F W H M_{2}}{2}$

where $\mathrm{FWHM}_{1}, \mathrm{FWHM}_{2}, \mathrm{~T}_{1}$, and $\mathrm{T}_{2}$ denote the first pulse width, the second pulse width, the position of the first peak, and the position of the second peak. This means that both recovered peaks will be registered for all algorithms.

\section{COMPARISON BETWEEN THE THREE ALGORITHMS AND DISCUSSION}

In this section, we present comparison between the underlined algorithms. This comparison is based on the input pileup peaks and the recovered two peaks. The error is found by the following relation

Error $=O_{i}-S_{i}$

where $\mathrm{O}_{\mathrm{i}}, \mathrm{S}_{\mathrm{i}}$, and i denote the input pileup peaks, the sum of the recovered peaks, and the set of data, respectively. In order to represent the accuracy and validity of these algorithms, comparison between both the input pileup and the recovered peaks is made. Figures 14-16 (a) represent the difference between the original overlapped peaks and the sum of recovered peaks for the three algorithms, respectively. On other hand, Figures 14-16 (b) show the error between 
the original overlapped peaks and the recovered peaks for the underlined three algorithms respectively. Furthermore, the input and estimated parameters of both the input pileup and recovered peaks for the three algorithms is shown in Table 2. Also, the estimated parameters error of the recovered two peaks for the considered three algorithms is depicted in Table 3. From these results we notice that the least square method has high accuracy for identifying both peak height and position of the overlapping peaks than other algorithms. However, the pulse width error is high to some extent. The first algorithm introduces the best recovered pulse width result. Also, the main strength of the proposed algorithm is the capability of resolving the original peaks in noisy environment. However, the first one introduces better results in clean environment. Therefore, when it combined with noise cancellation procedure that presented in our previous work [7], it overcomes this problem. From computational point of view, the third algorithm shows significant run time improvement compared with other two algorithms as shown in Table 2 . The number of events that fall within any one channel will vary in proportion to its width. The content of a typical channel varies inversely with the total number of channels provided over the spectrum. The channels content with smaller number of channels is larger than that with larger number of channels. Moreover, from Fig. 17, we notice that the resolution enhances with increases the number of channels. As the number of channels increases, the FWHM decreases. Resolution represents the FWHM with respect to position peak of the centroid. Consequently, good resolution is obtained. Therefore, resolution enhancement is an essential step especially at small number of channels.
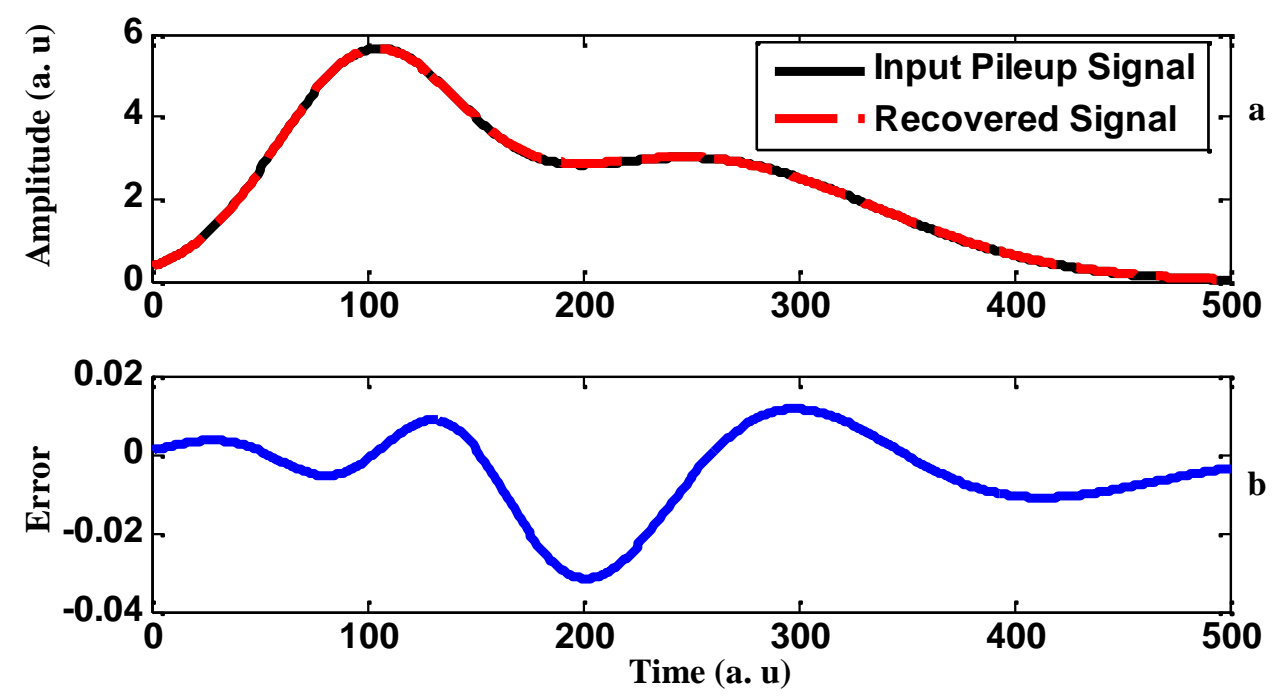

Figure 14. Accuracy of the direct search algorithm a) The input pileup peaks and summation of the recovered two overlapping peaks and b) The error that represents difference between the two signals 

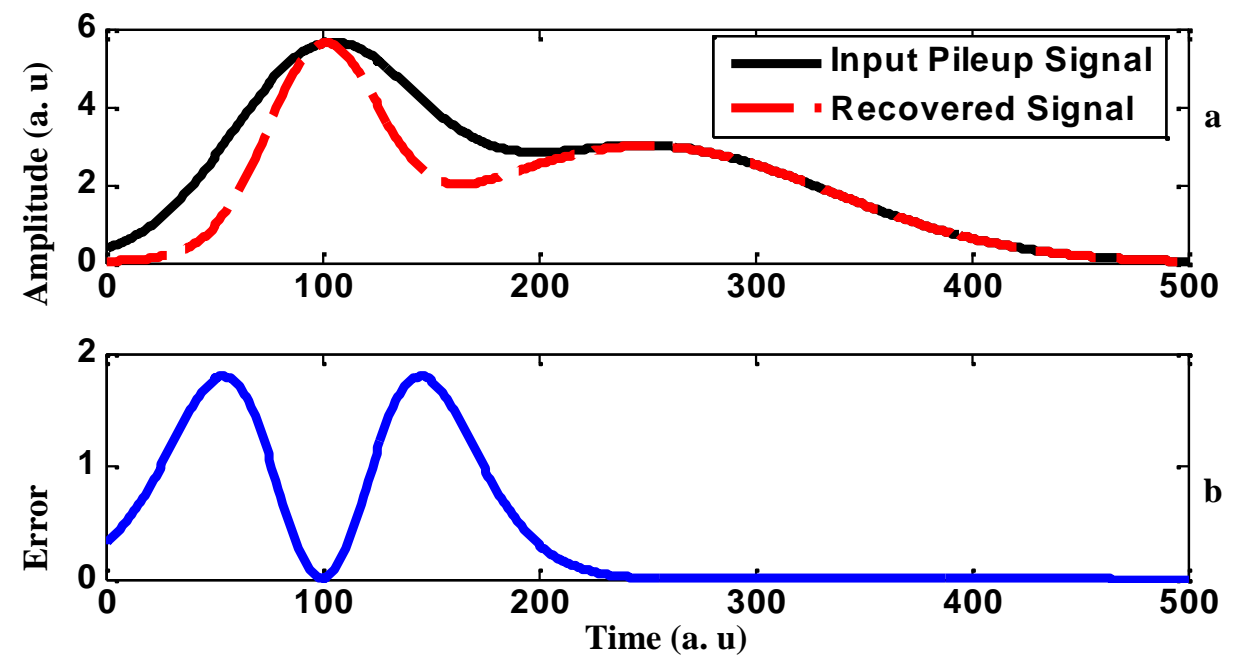

Figure 15. Accuracy of the least square algorithm a) The input pileup peaks and summation of the recovered two overlapping peaks and $b$ ) The error that represents difference between the two signals
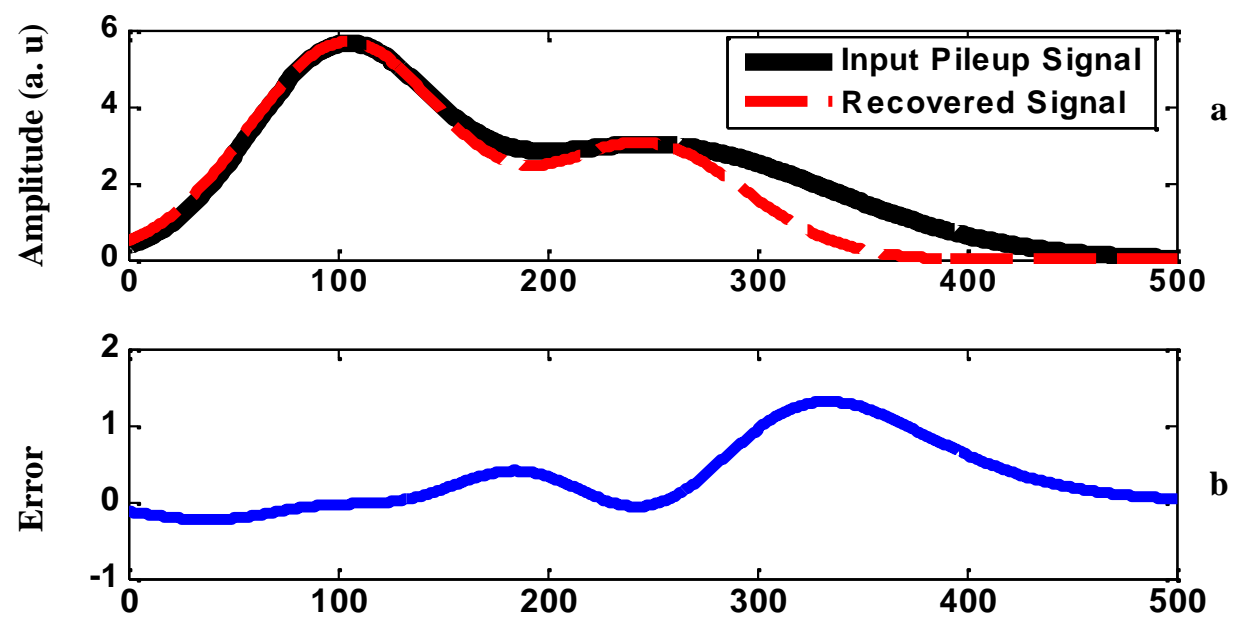

Figure 16. Accuracy of the maximum peak search algorithm a) The input pileup peaks and summation of the recovered two overlapping peaks and b) The error that represents difference between the two signals

Table 2. Input and estimated parameters of both the input and recovered peaks

\begin{tabular}{|c|c|c|c|c|c|c|}
\hline \multirow{2}{*}{} & \multicolumn{2}{|c|}{ Peak Height } & \multicolumn{2}{c|}{ Position } & \multicolumn{2}{c|}{ Width } \\
\cline { 2 - 7 } & $\begin{array}{c}1^{\text {st }} \\
\text { Peak }\end{array}$ & $\begin{array}{c}2^{\text {nd }} \\
\text { Peak }\end{array}$ & $\begin{array}{c}1^{\text {st }} \\
\text { Peak }\end{array}$ & $\begin{array}{c}2^{\text {nd }} \\
\text { Peak }\end{array}$ & $\begin{array}{c}1^{\text {st }} \\
\text { Peak }\end{array}$ & $\begin{array}{c}2^{\text {nd }} \\
\text { Peak }\end{array}$ \\
\hline $\begin{array}{c}\text { Input parameters } \\
\text { Estimated Value of Direct Search } \\
\text { algorithm }\end{array}$ & 5.0127 & 3 & 100 & 250 & 100 & 200 \\
\hline $\begin{array}{c}\text { Estimated value of Least Square } \\
\text { Algorithm }\end{array}$ & 5 & 3 & 100 & 250 & 60.06 & 120.1 \\
\hline $\begin{array}{c}\text { Estimated Value of Maximum Peak } \\
\text { Search Algorithm }\end{array}$ & 5.6642 & 3.0107 & 104 & 247 & & \\
\hline
\end{tabular}


Table 3. Estimated parameters errors of the underlined three algorithms

\begin{tabular}{|c|c|c|c|c|}
\hline Parameters Error & $\begin{array}{c}\text { Height } \\
\text { Error }\end{array}$ & $\begin{array}{c}\text { Position } \\
\text { Error }\end{array}$ & $\begin{array}{c}\text { Width } \\
\text { Error }\end{array}$ & $\begin{array}{c}\text { Run Time } \\
(\mathrm{ms})\end{array}$ \\
\hline Direct Search algorithm & 0.0024 & 0.0006 & 0.0005 & 93 \\
\hline Least Square Algorithm & 0 & 0 & 0.3994 & 484.8500 \\
\hline $\begin{array}{c}\text { Maximum Peak Search } \\
\text { Algorithm }\end{array}$ & -0.6645 & -0.0110 & 0.2244 & 31.2500 \\
\hline
\end{tabular}

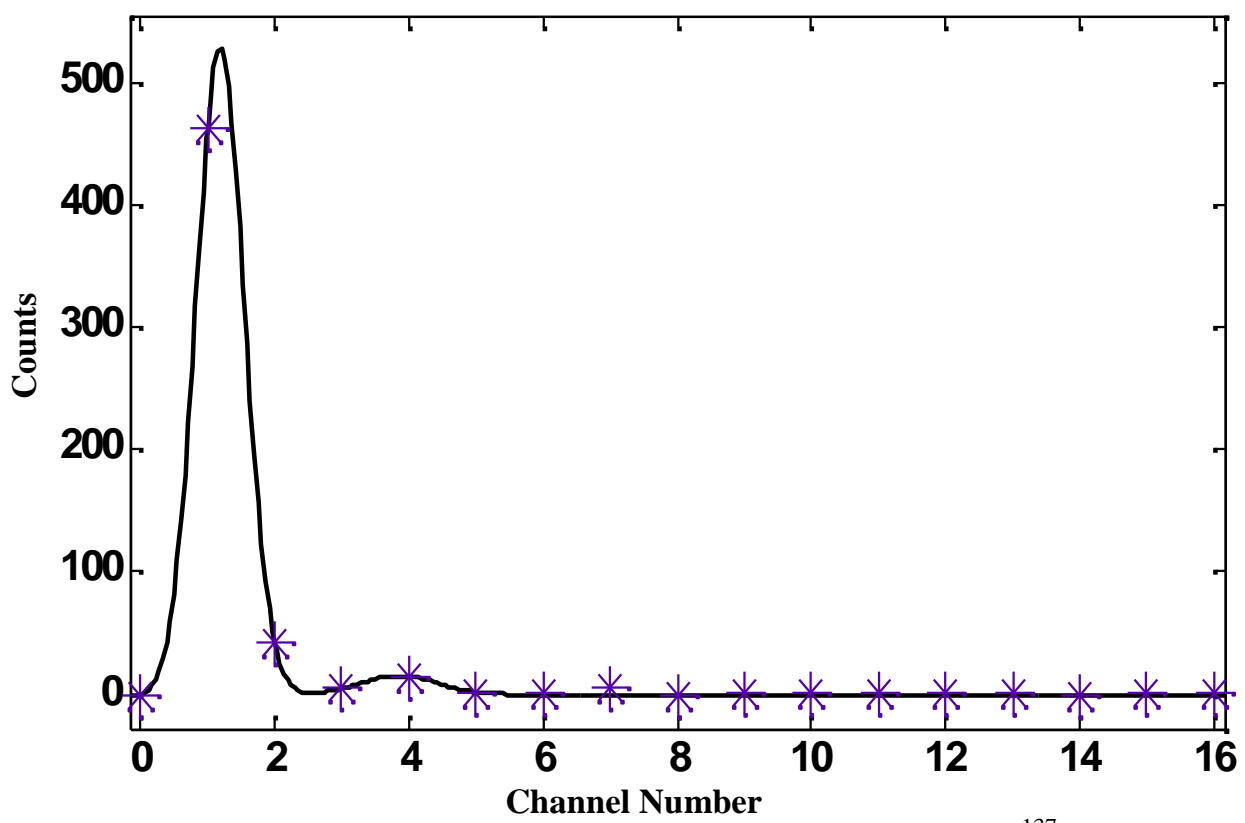

Figure 17. Channel content versus channel number for $\mathrm{Cs}^{137}$ source

\section{Conclusions}

Algorithms for overcoming the pileup problem of gamma-ray spectroscopy are presented. Three different algorithms are studied for pileup recovery. The first one is a direct search algorithm based on Nelder-Mead technique for determining both the peak position and peak amplitude. The second algorithm is the least square fitting method that is used to accurately determine the peak height and position of the overlapping peaks. The third algorithm is a proposed one which based on the first derivative of the signal combined with maximum peak search algorithm. This approach uses first derivatives for observing peak locations of pulses. Then, a direct search method for determining the pulse parameters such as position, maximum amplitude, pulse width and area are performed. The accuracy of the three algorithms is determined in terms of fitting accuracy and parameters error calculation of these algorithms. The direct search and maximum peak search algorithms proved to be satisfactorily robust against increasing level of noise. The direct search algorithm showed sensitivity to the level of noise. But, when it combined with noise cancellation procedure it overcomes this problem. From computational point of view, the first derivative combined with maximum peak search algorithm shows significant speed improvement compared with other two algorithms. From the obtained results, the least square fitting method gives better results for both peak height and position than other algorithms. However, the pulse width remains the main obstacle of this algorithm. 


\section{ACKNOWLEDGEMENTS}

This work is supported in part by IAEA research contract project (16409/RO).

\section{REFERENCES}

[1] S. M. Robinson, S. D. Kiff, E. D. Ashbaker, E. Flumerfelt, and M. Salvitti, (2009) "Effects of high count rate and gain shift on isotope-identification algorithms", Nuclear Instruments and Methods in Physics Research A, Vol. 610, pp. 509-514.

[2] C. J. Sullivan, S. E. Garner, K. B. Blagoev, and D. L. Weiss, (2007) "Generation of customized wavelets for the analysis of $\gamma$-ray spectra", Nuclear Instruments and Methods in Physics Research A, Vol. 579, pp. 275-278.

[3] W. Guo, S. H. Lee, and R. P. Gardner, (2004) "The Monte Carlo approach MCPUT for correcting pileup distorted pulse-height spectra", Nuclear Instruments and Methods in Physics Research A, Vol. 531, pp. 520-529.

[4] R. P. Gardner and S. H. Lee, (1999) "Monte Carlo simulation of pulse pileup", JCPDSInternational Centre for Diffraction Data, pp. 941-950.

[5] M. Bolic, and V. Drndarevi, (2002) "Digital gamma-ray spectroscopy based on FPGA technology", Nuclear Instruments and Methods in Physics Research A, Vol. 482, pp.761-766.

[6] W. Guo, R. P. Gardner, and F. Li, (2005) "A Monte Carlo code for simulation of pulse pileup spectral distortion in pulse-height measurement", International Centre for Diffraction Data, Advances in X-ray Analysis, Vol. 48, pp. 246- 252.

[7] M. S. El_Tokhy, I. I. Mahmoud, and H. A. Konber, (2010) "Comparative analysis between different linear filtering algorithms of gamma ray spectroscopy", Proceedings of the International Conference on Circuits, Systems, Signals, Malta.

[8] M. Morhac, and V. Matousek, (2009) "Complete positive deconvolution of spectrometric data", Digital Signal Processing Journal, Vol. 19, pp. 372-392.

[9] G. F. Knoll, (2000) "Radiation detection and measurement", Third Edition John Wiley and Sons, NY.

[10] W. Guo, R. P. Gardner, and C. W. Mayo, (2005) "A study of the real-time deconvolution of digitized waveforms with pulse pileup for digital radiation spectroscopy", Nuclear Instruments and Methods in Physics Research Section A,

Vol. 544, No. 3, pp. 668-678.

[11] MATLAB Help, the Language of Technical Computing, Version 7.0.0.19920 (R14), the Math Works, Inc., Copyright 1984-2004.

[12] R. Micchael Lewis, V. Torczon, and M. W. Trosset, (2011) "Direct search methods: then and now", http://www.cs.wm.edu/ va/research/jcam.pdf.

[13] J. C. Meza and M. L. Martinez, (1994) "On the use of direct search methods for the molecular conformation problem", Journal of Computational Chemistry, Vol. 15, No. 6, pp. 627-632.

[14] Chapter 8, Numerical Optimization, (2011) "Nelder-Mead method", http://math.fullerton.edu/mathews/n2003/neldermead/NelderMeadProof.pdf.

[15] T. O'Haver, (2011) "An introduction to signal processing in chemical analysis", http://terpconnect.umd.edu/ toh/spectrum/CurveFittingC.html.

[16] Jeffrey C. Lagarias, Jeffrey C. Lagarias, James A. Reeds, James A. Reeds, Margaret H. Wright, Margaret H. Wright, Paul E. Wright, and Paul E. Wright, (1996) "Convergence properties of the Nelder-Mead simplex algorithm in low dimensions", SIAM Journal of Optimization.

[17] Emma Suckling, (2011) "Introduction to gamma-ray spectroscopy using a thallium activated sodium iodide detector", http://www.emma-suckling.co.uk/work/MSc/Lab2a.pdf. 
[18] Peter J. Statham, (1977) "Pileup rejection: limitations and corrections for residual errors in energy-dispersive spectrometers", X-Ray Spectrometry, Vol. 6, No. 2.

[19] Yuntao Feng, (2006) "A Monte Carlo simulation and deconvolution study of detector response function for small field measurements", Doctorate of Philosophy (Ph.D.), Nuclear and Radiological Engineering of the College of Engineering, University of Cincinnati.

[20] New Interactive Calibration Demo, (2011) "FitzPeaks gamma analysis and calibration software", http://www.jimfitz.demon.co.uk/fitzpeak.htm.

[21] A. Likar and T. Vidmar, (2003) "A peak-search method based on spectrum convolution", J. Phys. D: Appl. Phys., Vol. 36, No. 1903, 1903, doi: 10.1088/0022-3727/36/15/323.

[22] M. W. Raad, and L. Cheded, (2011) "Efficient algorithms for pulse parameter estimation, pulse peak localization and pileup reduction in gamma ray spectroscopy", http://faculty.kfupm.edu.sa/COE/raad/publications/imtc2007.pdf.

[23] G. Ripamonti, A. Geraci, (1996) "Fast least mean squares method for time determination and pileup rejection in high resolution spectroscopy", Nuclear Science Symposium, IEEE, Vol.1, pp. 474- 477.

[24] V. B. Zlokazov, (1982) "Method for an automatic peak search in gamma-ray spectra", Nuclear Instruments and Methods in Physics Research Vol. 199, No. 3, pp. 509-519.

[25] A. Likar and T. Vidmar, (2003) "A peak-search method based on spectrum convolution", Journal of Physics D: Applied Physics, Vol.36, No. 15.

[26] Markku J. Koskelo and Mark T. Mercier, (1990) "Verification of gamma spectroscopy programs: a standardized approach", Nuclear Instruments and Methods in Physics Research Section A: Accelerators, Spectrometers, Detectors and Associated Equipment, Vol. 299, No. 1-3, pp. 318-321.

[27] A. H. Lawrence, and R. A. Goubran, (1991) "Experimental signal analysis in ion mobility spectrometry", International Journal of Mass Spectrometry and Ion Processes, Vol. 104, No. 3, pp. 163-178.

[28] https://ftirsearch.com/help/algo.htm\#First Derivative Least Squares Search.

[29] H. Holden, and E. LeDrew, (1998) "Deconvolution of measured spectra based on principal components analysis and derivative spectroscopy", Geoscience and Remote Sensing Symposium Proceedings (IGARSS '98), IEEE International, Seattle, WA, Vol. 2, pp. 760-762. 